\title{
Improvement of GPR SAR-based techniques for accurate detection and imaging of buried objects
}

\author{
Marcos González-Díaz, María García-Fernández, Student Member, IEEE, \\ Yuri Álvarez-López, Senior Member, IEEE and Fernando Las-Heras, Senior Member, IEEE
}

\begin{abstract}
This contribution introduces three methodologies to improve Synthetic Aperture Radar (SAR)-based techniques used in Ground Penetrating Radar (GPR) systems. They consist of i) equalization of the frequency response of the transmitting $(T x)$ and receiving $(R x)$ antennas, ii) processing of SAR images using partially overlapped frequency sub-bands, and iii) imaging domain clustering. The goal is to combine ground penetration capabilities of lower frequency bands with the resolution achieved when increasing the overall frequency band, resulting in enhanced detection and imaging capabilities. Validation of these techniques has been done at three levels: first using simulations, next by means of measurements in a controlled scenario and, finally, using a portable setup deployed in a realistic scenario.
\end{abstract}

Index Terms-Ground Penetrating Radar (GPR), subsurface sensing and imaging, Synthetic Aperture Radar (SAR).

\section{INTRODUCTION}

$\mathbf{G}$ ROUND Penetrating Radar (GPR) is a non-invasive technique for subsurface sensing and imaging applications, which is based on the transmission and reception of electromagnetic waves using a radar system [1]. Transmitted radar waves are reflected on the interfaces between two media with different constitutive parameters (namely conductivity and permittivity). GPR exhibits several advantages over other Non-Destructive Testing (NDT) techniques (such as metal detectors, thermal cameras, or ultrasounds) for the detection of buried targets. Among others, it can be cited: i) capability of detecting both metallic and non-metallic objects, ii) possibility of obtaining high-resolution images of the ground and the buried targets, and iii) use of a mature, low-cost technology.

GPR has been extensively applied in several fields, such as inspection of civil infrastructure (e.g. bridges) [2], [3], location of underground tunnels [4], and landmine detection [5], [6]. In the latter, a novel technology based on an airborne GPRSynthetic Aperture Radar (SAR) system has been developed, thanks to the advances in accurate positioning and georeferring sensors (such as Real Time Kinematics, RTK, and laser rangefinders) [7], [8], [9].

M. González-Díaz, M. García-Fernández, Y. Álvarez-López, and F. LasHeras are with the Area of Signal Theory and Communications of the University of Oviedo. Gijón (Asturias), 33203 Spain e-mail: garciafmaria@uniovi.es

This work has been supported by the Ministerio de Educación - Gobierno de España under grant FPU15/06341; by the Ministerio de Ciencia, Innovación y Universidades - Gobierno de España under project RTI2018-095825B-I00 ("MilliHand"); by the Government of the Principality of Asturias (PCTI) and European Union (FEDER) under grants IDI/2018/000191 and IDI/2017/000095; and by the Instituto Universitario de Investigación Industria de Asturias (IUTA), under projects SV-17-GIJON-1-18, SV-18-GIJON-1-07 ("Georradar embarcado en un dron para detección de minas y otros objetos enterrados (GEODRON)"), and SV-19-GIJON-1-17.

Manuscript received November 12, 2018. Accepted July 4, 2019.
GPR image resolution depends on: i) the frequency bandwidth $(\triangle B W)$, which determines range or depth resolution $\Delta R=v_{\text {prop }} /(2 \Delta B W)$, being $v_{\text {prop }}$ the speed of light in a particular medium, and ii) the GPR antenna beamwidth, which is related to cross-range or lateral resolution. The latter can be improved by means of SAR techniques [10], based on the coherent combination of a set of measurements taken along a certain path (as done, for example, in airborne-based SAR systems [7], [8], [9], [11]). These measurements have to be accurately geo-referred so that the phase error due to position uncertainty does not distort the resulting image [8]. Although GPR-SAR-based techniques are not capable of recovering both the profile and constitutive parameters as accurately as inverse scattering techniques [12], [13], the former have much less computational complexity and are more robust against measurement uncertainties.

GPR-SAR images can also be improved (e.g. reducing false echoes and artifacts, improving the focusing) by taking into account the different wave velocity in the air and in the ground. This requires the knowledge of the ground constitutive parameters [14]. In this sense, several GPR-SAR techniques have been developed for microwave imaging of the ground and the objects buried in it. Among them, it can be cited:

1) Delay-And-Sum (DAS): based on ray-tracing, it is one of the most extended techniques due to its ease of implementation [15].

2) Phase Shift Migration (PSM): also known as $\omega-k$ migration. It is based on the simplification of the electromagnetic wave equation, taking into account the constitutive parameters of the ground [16], [17].

3) Piecewise Synthetic Aperture Radar (P-SAR): a modified version of PSM that takes into account the reflection and transmission coefficients of electromagnetic waves when passing through the different layers of the ground [18].

4) Wiener filter-based GPR-SAR: this technique is based on Green functions for layered media [18], [19].

Several methodologies have been developed to enhance the quality of GPR images. For example, those ones conceived to mitigate the clutter due to the air-ground reflection, such as Singular Value Decomposition (SVD)-based projection methods [20], [21], time-gating [22], and average subtraction [21], [22].

\section{A. Aim and scope}

Novel GPR systems such as the one described in [8] require more robust GPR-SAR processing techniques capable 
of providing high resolution images while keeping computational cost low. The use of cheap, off-the-shelf radiofrequency hardware allows reducing the overall cost of the GPR system, but at the expense of worsening the performance with respect to the use of GPR-specific hardware. For example, Ultra Wideband (UWB) antenna parameters such as directivity or return losses may vary significantly within the working frequency band, thus requiring these parameters to be taken into account in GPR-SAR processing techniques. Besides, UWB GPR systems allow increasing resolution and hence the capability of detecting smaller targets. However, constitutive parameters have a strong dependence with frequency, resulting in worse penetration as frequency increases. Efficient postprocessing techniques for UWB GPR systems that make use of a proper characterization of the frequency response of the implemented GPR system have not been extensively developed in the current state-of-the-art of GPR techniques.

Aiming to improve the resolution of GPR-SAR images, to reduce clutter and artifacts, and to speed-up processing time, this contribution introduces three novel GPR-SAR processing techniques:

1) Equalization of the frequency response of the $T x$ and $R x$ antennas, so that the contributions of the scattered field at different frequencies have similar weight in the GPR-SAR image.

2) GPR-SAR image processing in sub-bands. The goal is to take advantage of lower frequency bands, which exhibit better penetration capabilities, and upper frequency bands, where resolution is better because of the larger electric size of the synthetic aperture.

3) Imaging domain partitioning, in order to take into account the $\mathrm{Tx} / \mathrm{Rx}$ antenna beamwidth at different working frequencies. The idea is to backpropagate, for each frequency, the scattered field to those clusters within the antenna beamwidth, thus minimizing reflections from nondesired contributions and speeding up the processing.

These techniques have been proposed to improve the airborne-based GPR-SAR system described in [8], where detection of non-metallic targets (e.g. Improvised Explosive Devices, IEDs, mainly composed by dielectric materials) is one of the target applications of the system. Nevertheless, these techniques are perfectly suitable for any GPR-SAR system.

The paper is structured as follows: Section II presents an overview of GPR-SAR-based techniques, focusing on DAS and PSM. The three methodologies introduced to improve GPR-SAR are explained in Section III. Validation by means of simulations and measurements (in laboratory and in a realistic scenario) is described in Section IV. Finally, conclusions are drawn in Section V.

\section{GPR-SAR TECHNIQUES}

As mentioned before, taking into account the constitutive parameters of the soil in GPR-SAR processing techniques improves range resolution and helps to reduce the clutter. Thus, the techniques described in this Section are expressed in their multilayer form. If the soil permittivity cannot be estimated, it can be assumed a homogeneous medium (e.g. free-space propagation with $\varepsilon_{r}=1$ ).

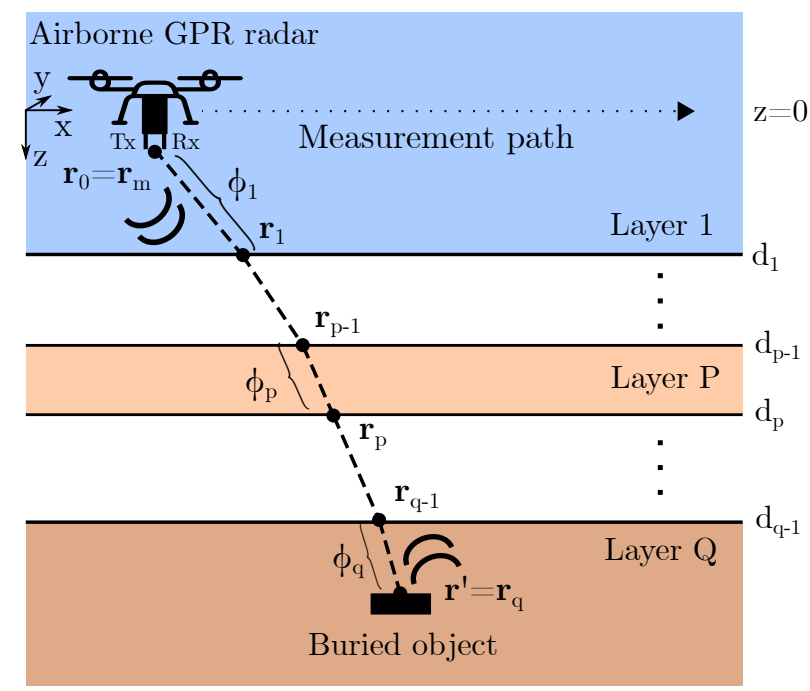

Fig. 1. General scheme of a multilayer evaluation for GPR-SAR techniques.

In addition to these GPR-SAR techniques, several preprocessing methods mentioned in Section I can also be applied in order to reduce echoes coming from the impedance mismatching between the air and the ground. Average subtraction [22] can be used as long as the set-up measurement conditions are favorable for its implementation.

\section{A. Delay-And-Sum (DAS)}

The basic principle of DAS GPR-SAR imaging is the coherent combination of the backpropagated scattered field collected on $M$ acquisition points at $N$ frequencies, $E_{\text {scatt }}\left(\boldsymbol{r}_{m}, f_{n}\right)$. Assuming a monostatic or quasi-monostatic configuration (i.e. $\mathrm{Tx}$ and $\mathrm{Rx}$ placed practically at the same position), the reflectivity at a single point $\rho_{Q}\left(\boldsymbol{r}^{\prime}\right)$ in the $\mathrm{Q}$ layer (Fig. 1) can be calculated as follows (Eq. 1):

$$
\rho_{Q}\left(\boldsymbol{r}^{\prime}\right)=\sum_{m=1}^{M} \sum_{n=1}^{N} E_{\text {scatt }}\left(\boldsymbol{r}_{m}, f_{n}\right) \prod_{p=1}^{q} e^{+j 2 \phi_{p, n}}
$$

where $\boldsymbol{r}_{m}$ is the position of the $m$-th acquisition point, $f_{n}$ is the $n$-th frequency and $\phi_{p, n}$ are the phase-shifts due to the wave propagation in the $p$-th layer, as depicted in Fig. 1. These terms are defined in Eq. 2:

$$
\phi_{p, n}=k_{p, n} \cdot\left\|\boldsymbol{r}_{p}-\boldsymbol{r}_{p-1}\right\|_{2}
$$

where $k_{p, n}$ is the wavenumber in the $p$-th layer for the $n$ th frequency, $\boldsymbol{r}_{p}$ (for $p=1, \ldots, q-1$ ) is the refraction point at the $p$-th layer and $\boldsymbol{r}^{\prime}=\boldsymbol{r}_{q}$ is one of the points where the reflectivity is calculated (at layer Q), as indicated in Fig. 1. The refraction point can be derived from Snell's law solving a 4th order equation. However, in order to reduce complexity and computational time, it is estimated using the approximation method proposed in [15].

This approximation method is expressed in Eq. 3 for a two-layer scenario as depicted in Fig. 2 (a), where $d_{p}$ are distances in z-axis referred to the antenna position (e.g. $d_{1}$ is the distance from the interface between $\varepsilon_{r, 1}-\varepsilon_{r, 2}$ to the 


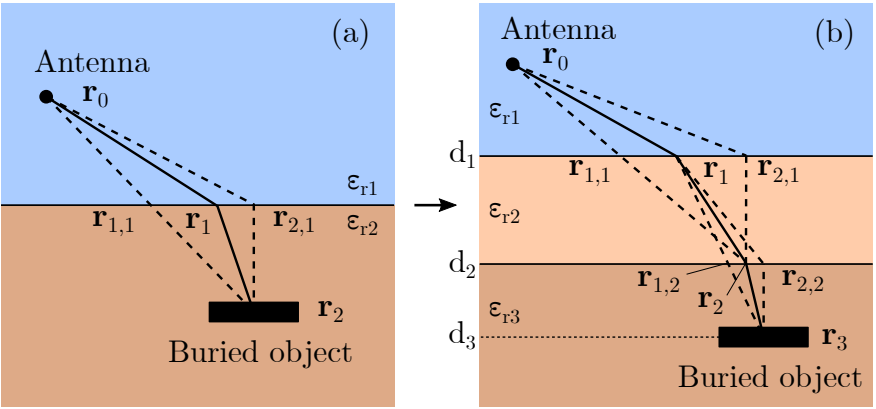

Fig. 2. Refraction point estimation method: two layers (a) and three layers (b).

antenna). As DAS algorithm has been defined in its multilayer form, the refraction point at each layer is calculated by an extension of the approximation method given in [15]. This extension consists of solving the equation system derived from the previous two layer definition. A scheme of the refraction points of a typical three layer scenario is shown in Fig. 2 (b). After solving the equation system, refraction points for the three-layer scenario are given by Eq. 4, where $\widetilde{\boldsymbol{r}_{p}}=\boldsymbol{r}_{p}-\boldsymbol{r}_{0}=\left(\widetilde{x_{p}}, \widetilde{y_{p}}, d_{p}\right)=\left(x_{p}-x_{0}, y_{p}-y_{0}, d_{p}\right)$ and $\Delta_{1,2}, \Delta_{2,3}$ are given by Eq. 5 .

$$
\begin{gathered}
\boldsymbol{r}_{1}=\boldsymbol{r}_{2,1}+\sqrt{\frac{\varepsilon_{r 1}}{\varepsilon_{r 2}}}\left(\boldsymbol{r}_{1,1}-\boldsymbol{r}_{2,1}\right) \\
\left\{\begin{array}{l}
\widetilde{\boldsymbol{r}_{1}}=\left(\Delta_{1,2} \widetilde{x_{2}}, \Delta_{1,2} \widetilde{y_{2}}, d_{1}\right) \\
\widetilde{\boldsymbol{r}_{2}}=\left(\Delta_{2,3} \widetilde{x_{3}}, \Delta_{2,3} \widetilde{y_{3}}, d_{2}\right)
\end{array}\right. \\
\left\{\begin{array}{l}
\Delta_{1,2}=1+\sqrt{\frac{\varepsilon_{r 1}}{\varepsilon_{r 2}}}\left(\frac{d_{1}-d_{2}}{d_{2}}\right) \\
1+\sqrt{\frac{\varepsilon_{r 2}}{\varepsilon_{r 3}}} \frac{d_{2}-d_{3}}{d_{3}-d_{1}} \\
\Delta_{2,3}=\frac{\left(d_{3}\right)\left(d_{1}-d_{2}\right)}{1-\sqrt{\frac{\varepsilon_{r 2}}{\varepsilon_{r 3}}} \frac{d_{3}-d_{2}}{d_{3}-d_{1}}-\sqrt{\frac{\varepsilon_{r 1}}{\varepsilon_{r 3}}} \frac{d_{3}-d_{2}}{d_{2}\left(d_{3}-d_{1}\right)}}
\end{array}\right.
\end{gathered}
$$

\section{B. Phase Shift Migration algorithm (PSM)}

PSM algorithm also combines coherently the backpropagated scattered field. However, unlike DAS algorithm, it is based on the wave equation and the Exploding Reflector Model (ERM) from the seismic field [23]. In particular, it assumes that the medium is practically homogeneous along $X Y$ plane and the EM waves are generated by each point in the investigation domain rather than by the antennas. Therefore, the propagation velocity is assumed to be half its true value. In order to speed up calculations, the backpropagated scattered field is calculated in the $f-k$ domain. Hence the reflectivity at a single $X Y$ plane (denoted as $z^{\prime}$ plane) in the $Q$ layer $\rho_{Q}\left(z^{\prime}\right)$ is calculated as follows (Eq. 6):

$$
\rho_{Q}\left(z^{\prime}\right)=\sum_{n=1}^{N} \mathrm{~F}_{x y}^{-1}\left\{\begin{array}{r}
E_{\text {scatt }}\left(k_{x}, k_{y}, f_{n}\right) e^{+j \mathrm{~d}_{1} k_{z, 1, \mathrm{n}}} \\
\cdot\left[\prod_{p=2}^{q-1} e^{+j\left(\mathrm{~d}_{p}-\mathrm{d}_{p-1}\right) k_{z, p, n}}\right] \\
\cdot e^{+j\left(z^{\prime}-\mathrm{d}_{q-1}\right) k_{z, q, n}}
\end{array}\right\}
$$

where $\mathrm{d}_{p}$ is the $z$-distance from the interface between layers $p$ and $p+1$ to the antennas (see Fig. 1), $k_{x}, k_{y}$ are the wavenumber components in $x$ and $y$ directions, $k_{z, p, n}$ is the wavenumber component in $z$ direction at the $p$-th layer and $n$-th frequency, and $\mathrm{F}_{x y}\{\cdot\}$ is the Fourier Transform in the $x-y$ domain. The $k_{z, p, n}$ term is defined in Eq. 7 as:

$$
k_{z, p, n}=\sqrt{\frac{\left(2 \pi f_{n}\right)^{2}}{v_{p}^{2}}-k_{x}^{2}-k_{y}^{2}}
$$

where $v_{p}$ is half of the propagation velocity in the $p$-th layer.

As mentioned before, if the soil permittivity cannot be estimated, a homogeneous propagation velocity $v_{p}=\mathrm{c} / 2$ will be assumed.

\section{IMPROVEMENTS TO SAR TECHNIQUES}

With the purpose of obtaining high resolution GPR-SAR based images, an ultra wide frequency band from 0.1 to $6.5 \mathrm{GHz}$ has been considered. The reason of this choice is because it is the same as the working frequency band of the radar used for experimental validation [24]. However, taking advantage of the entire frequency bandwidth (using the two aforementioned GPR SAR processing techniques) becomes challenging because the propagation losses and the directivity of the transmitting and receiving antennas vary significantly with frequency. Thus, as mentioned in Section I, several methods are proposed in order to overcome these limitations.

\section{A. Equalization of the frequency response}

Two UWB Vivaldi antennas [25] have been selected for the implementation of the GPR-SAR system. These antennas exhibit a $S_{11}$ parameter below $-10 \mathrm{~dB}$ from 0.6 to $6 \mathrm{GHz}$. However, as observed in Fig. 3, their directivity fluctuates more than $7 \mathrm{~dB}$ along the working frequency band.

Fig. 4 shows the frequency response of the GPR system for a scenario where the antennas are $1 \mathrm{~m}$ above ground (normal incidence/reflection). Amplitude levels in the working frequency band exhibit a variation greater than $15 \mathrm{~dB}$ in addition to smaller multipath fluctuations. Consequently, SAR images are affected by these differences causing lower frequencies to be dominant over higher ones, thus reducing the effective bandwidth and, in consequence, the resolution. Hence, the equalization of the frequency response, which consists of normalizing the SAR image for each discrete frequency, is proposed to take advantage of the entire frequency band.

Equalization of the frequency response is done as follows: for each $n$-th frequency of the working frequency band, the reflectivity image is calculated and saved. For example, for the DAS algorithm, Eq. 1 is recasted as follows (Eq. 8):

$$
\rho_{Q}\left(\boldsymbol{r}^{\prime}, n\right)=\sum_{m=1}^{M} E_{\text {scatt }}\left(\boldsymbol{r}_{m}, f_{n}\right) \prod_{p=1}^{q} e^{+j 2 \phi_{p, n}}
$$

Then, each $n$-th reflectivity image is normalized with respect to the maximum of its absolute value. This operation mitigates the influence of the frequency response of the $\mathrm{Tx}$ and $\mathrm{Rx}$ antennas (Eq. 9): 


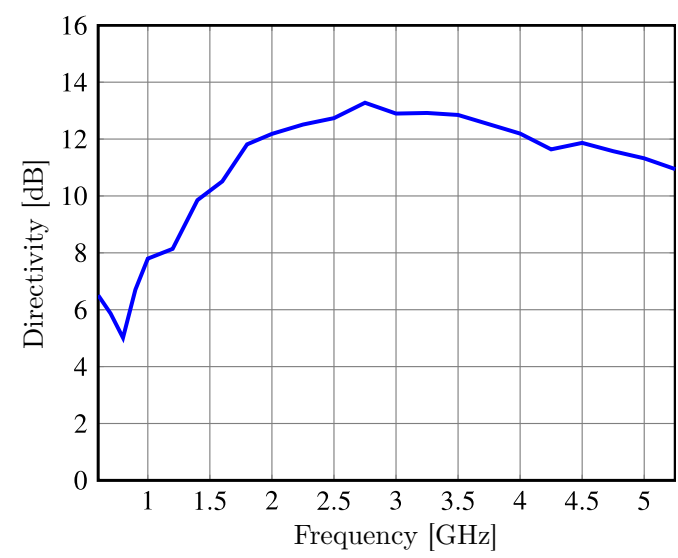

Fig. 3. Directivity of the Vivaldi antennas [25] from 0.3 to $5.4 \mathrm{GHz}$, calculated from radiation pattern integration. Radiation pattern was measured at spherical range in anechoic chamber.

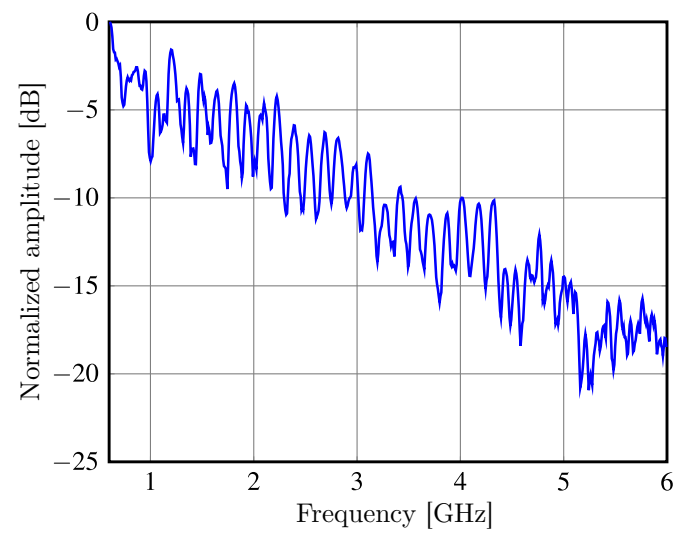

Fig. 4. Maximum amplitude at each frequency from a typical GPR scenario.

$$
\overline{\rho_{Q}\left(\boldsymbol{r}^{\prime}, n\right)}=\rho_{Q}\left(\boldsymbol{r}^{\prime}, n\right) / \max \left\{\left|\rho_{Q}\left(\boldsymbol{r}^{\prime}, n\right)\right|\right\}
$$

Finally, the $N$ reflectivity images are added.

\section{B. Sub-band processing}

The propagation losses of EM waves and the constitutive parameters of the soil are frequency dependent [26]. Moreover, as explained before, the antennas have different directivity along the entire frequency band, which affects SAR processing. As a consequence, depending on the characteristics of the analyzed scenario, a sub-band processing method is proposed. An upper frequency sub-band takes advantage of greater directivity, resulting in better cross-range resolution. On the other hand, lower frequency sub-bands decrease the cross-range resolution, but improve penetration capabilities. It should be taken into account that a low directivity leads to a loss of imaging quality due to reflections from non-desired contributions e.g. targets outside the area of interest.

\section{Imaging domain partitioning}

This technique uses the radiation pattern of Vivaldi antennas to increase signal to noise ratio and to reduce calculation

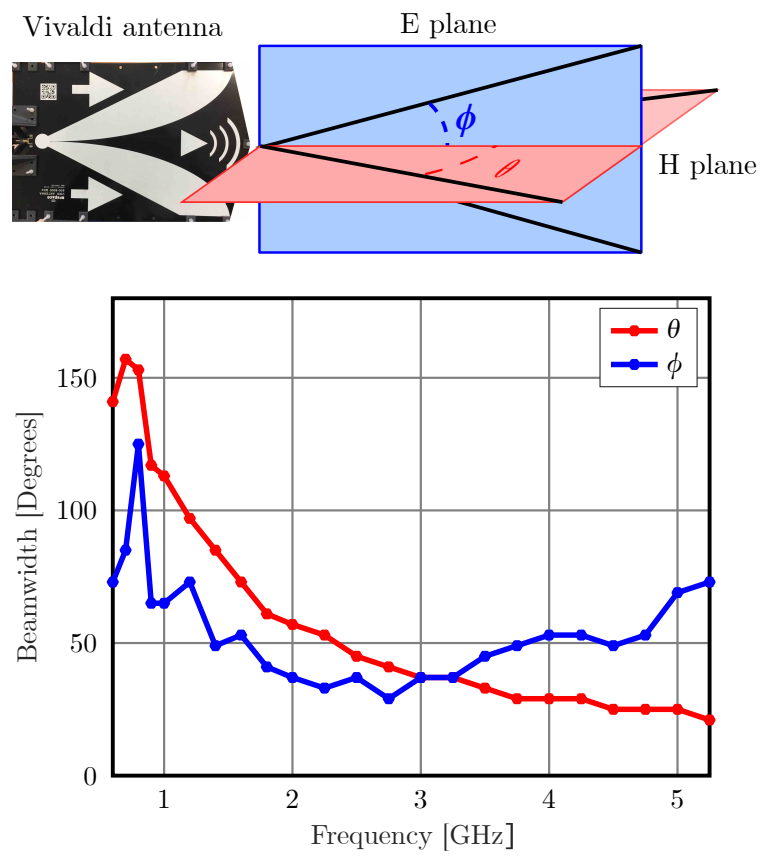

Fig. 5. Beamwidth of Vivaldi antenna [25] measured at spherical range in anechoic chamber. $H$ plane $(\theta)$ and $E$ plane $(\phi)$

time. It consists of dividing the imaging domain into several subdomains or cells, then combining only the backpropagated scattered field at the reconstruction cells selected for each frequency. The number of cells is based on the antenna beamwidth (Fig. 5), as depicted in Fig. 6. Since the backpropagated scattered field is not calculated in the entire domain but in the selected cells or subdomains, the overall computational cost is decreased.

The reconstruction domain is defined in Eq. 10, where $C_{x}$ and $C_{y}$ are the number of cells around the measurement position in the $x$ and $y$ direction for each frequency, respectively. Fig. 6 illustrates the proposed method, where $h$ is the distance between the transmitter and the ground, $\theta$ and $\phi$ are the beamwidths of the antenna at $E$ and $H$ planes, and $a, b, c$ are the $x, y$, and $z$ dimensions of the reconstruction cell. For the sake of simplicity, $a=\delta x, b=\delta y, c=\delta z$, where $\delta x, \delta y$ and $\delta z$ are the sampling intervals. In the case of $\delta x, \delta y$ (crossrange), these intervals are the same as the acquisition domain sampling rate. Sampling rate along range axis $(\delta z)$ is given by the theoretical range resolution, $\Delta R=v / 2 B W$, being $v$ the propagation velocity and $B W$ the bandwidth of the system.

$$
\left\{\begin{array}{l}
C_{x, n}=\frac{2 h \tan \left(\phi_{n} / 2\right)}{\mathrm{a}} \\
C_{y, n}=\frac{2 h \tan \left(\theta_{n} / 2\right)}{\mathrm{b}}
\end{array}\right.
$$

\section{SySTEM VALIDATION}

\section{A. Simulations}

Several scenarios were simulated using the open-source software gprMax [27]. In these scenarios, a 2-D scanning was 


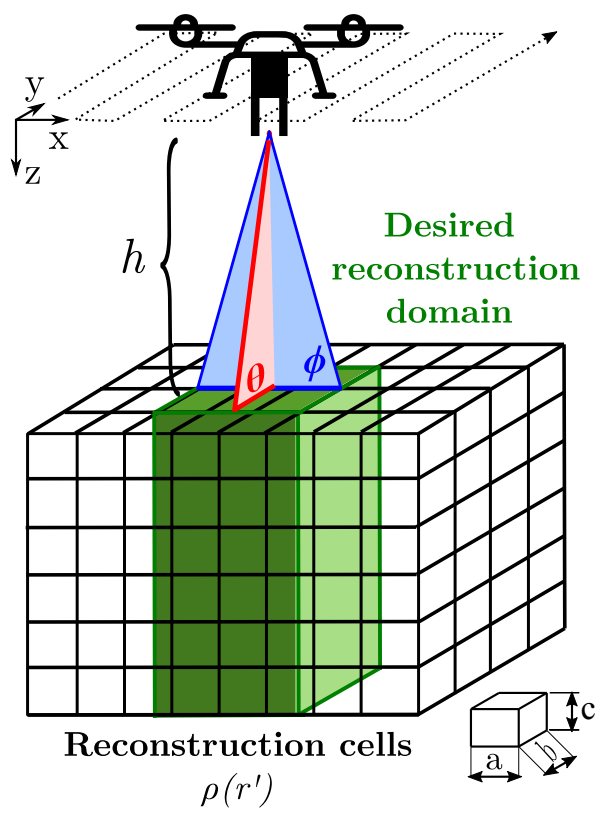

Fig. 6. Scheme of the desired reconstruction domain.

performed at $z=0 \mathrm{~cm}$ and $x \in(-24,24) \mathrm{cm}$ with a step-size of $2 \mathrm{~cm}$ (about $\lambda / 2$ ).

1) Analysis of GPR-SAR methods: The first part of this subsection is devoted to analyze the performance of the GPR-SAR methods (without improvements) and to show the influence of taking into account the soil constitutive parameters on the SAR images.

For these simulations, Hertzian dipoles arranged in a quasimonostatic configuration are used as transmitter and receiver antennas. The first derivative of a gaussian pulse has been selected as transmitted signal with a center frequency of 3 $\mathrm{GHz}$ and a bandwidth of approximately $3.5 \mathrm{GHz}$.

The first analyzed scenario (Scenario A) is depicted in Fig. 7. This is a favorable scenario for detecting buried objects due to the low conductivity (and thus low losses) of dry sand. Scenario A results are presented in Fig. 8. In order to interpret the different images, the differences between monolayer and multilayer processing techniques must be remarked. Monolayer techniques do not take into account the permittivity of the ground (assuming the whole scenario is a unique homogeneous medium, in particular with $\varepsilon_{r}=1$ ), whereas multilayer techniques consider different permittivities at each layer. Hence, both objects are displaced downwards and distorted after using the former techniques (Fig. 8 (a),(b)) whereas the objects appear at their actual depths using the latter ones (Fig. 8 (c),(d)). Furthermore, multilayer techniques have been complemented by the average subtraction method in order to remove reflections from the dry sand interface, as shown in Fig. 8 (c),(d). It can be noticed that the plastic object can be imaged with enough resolution to distinguish top and bottom sides. As expected, the metallic objects exhibits higher reflectivity than the plastic one.

Concerning PSM and DAS imaging techniques, it can be concluded that there are no remarkable differences in the SAR

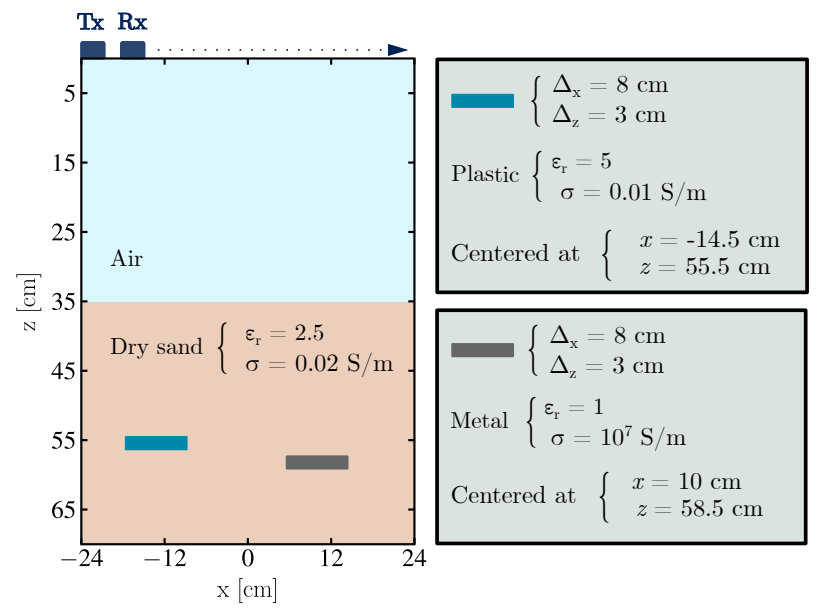

Fig. 7. Simulated Scenario A.
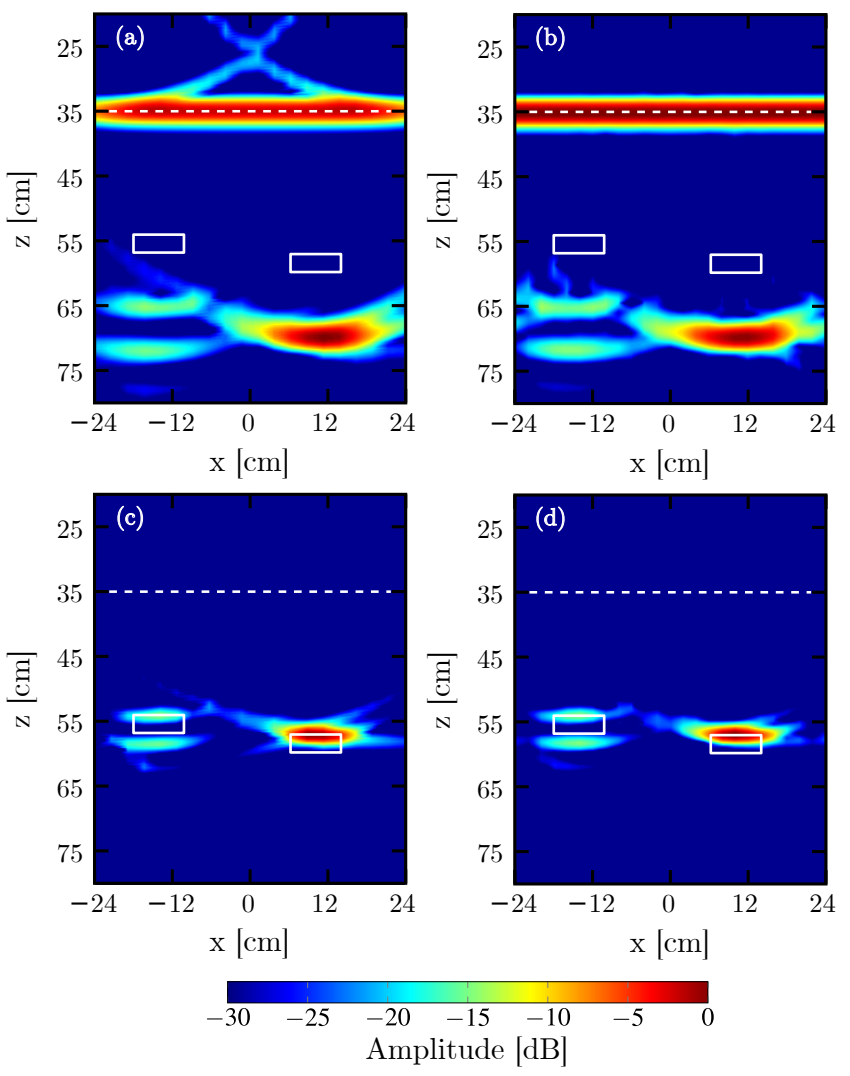

Fig. 8. Reflectivity images of Scenario A. (a) DAS monolayer. (b) PSM monolayer. (c) DAS multilayer. (d) PSM multilayer. Average subtraction method has been applied in (c),(d).

images, although PSM is about 30 to 50 times faster than DAS, thanks to the use of the FFTs (Fast Fourier Transforms).

In the second simulated scenario (Scenario B, see Fig. 9), the soil is composed by two layers of sand with different moisture levels. It must be noticed that the wetter the soil is, the greater the permittivity and conductivity are, thus being more difficult to detect buried targets. In this case, average subtraction method has not been applied in order to show that the air - dry sand and the dry sand - wet sand interfaces can be 


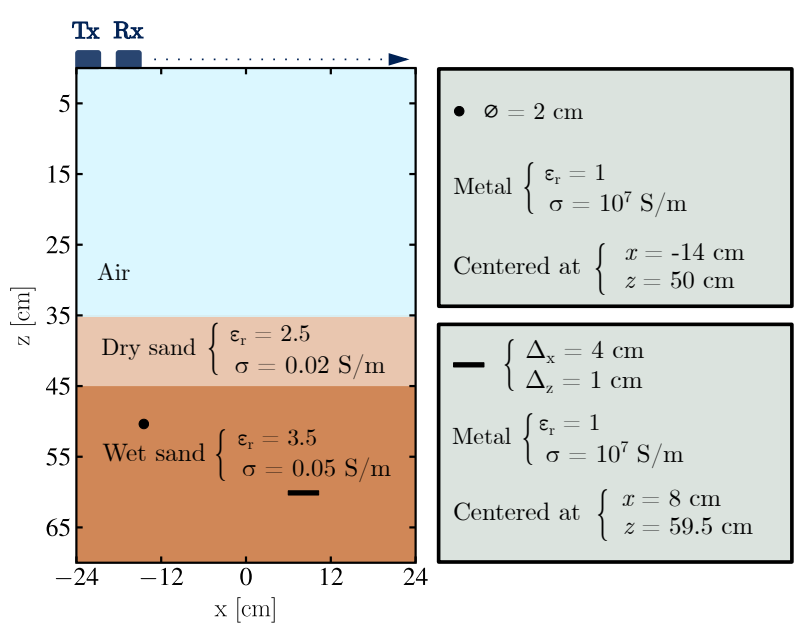

Fig. 9. Simulated Scenario B.

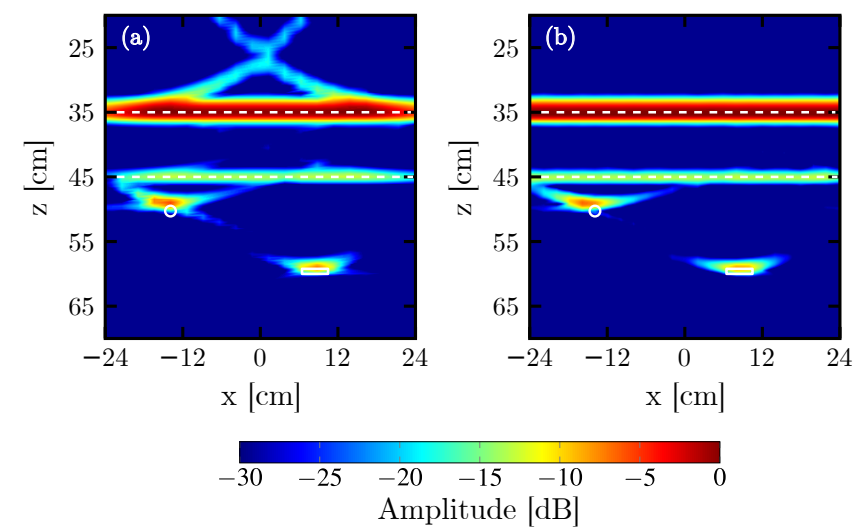

Fig. 10. Reflectivity images of Scenario B. (a) DAS multilayer. (b) PSM multilayer.

clearly distinguished. Since both metallic objects have enough contrast with the surrounding medium, not using average subtraction does not affect their detection. As in scenario A, both the position and the shape of the buried targets can be recovered (Fig. 10). Again, PSM and DAS imaging results are practically the same.

2) Preliminary analysis of improvements: The second part of this subsection is devoted to assess the effect of the proposed GPR-SAR improvements. As aforementioned, it must be noticed that these improvements are mainly needed due to frequency dependent behaviour of both the antennas and the soil.

In order to analyze the effect of the equalization of the frequency response, instead of ideal dipole antennas a pair of real bow-tie antennas (with central frequency of $1.5 \mathrm{GHz}$ ) have been used as Tx and Rx. The resulting SAR images obtained for DAS algorithm are shown in Fig. 11 with and without equalization (taken into account frequencies between 1 and $5 \mathrm{GHz}$ ). In general, an improvement in the range resolution can be noticed for both scenarios. In scenario A, the plastic object top interface is also better focused using equalization. In scenario B, the dry sand - wet sand interface is clearly distinguishable with equalization. However, if equalization is
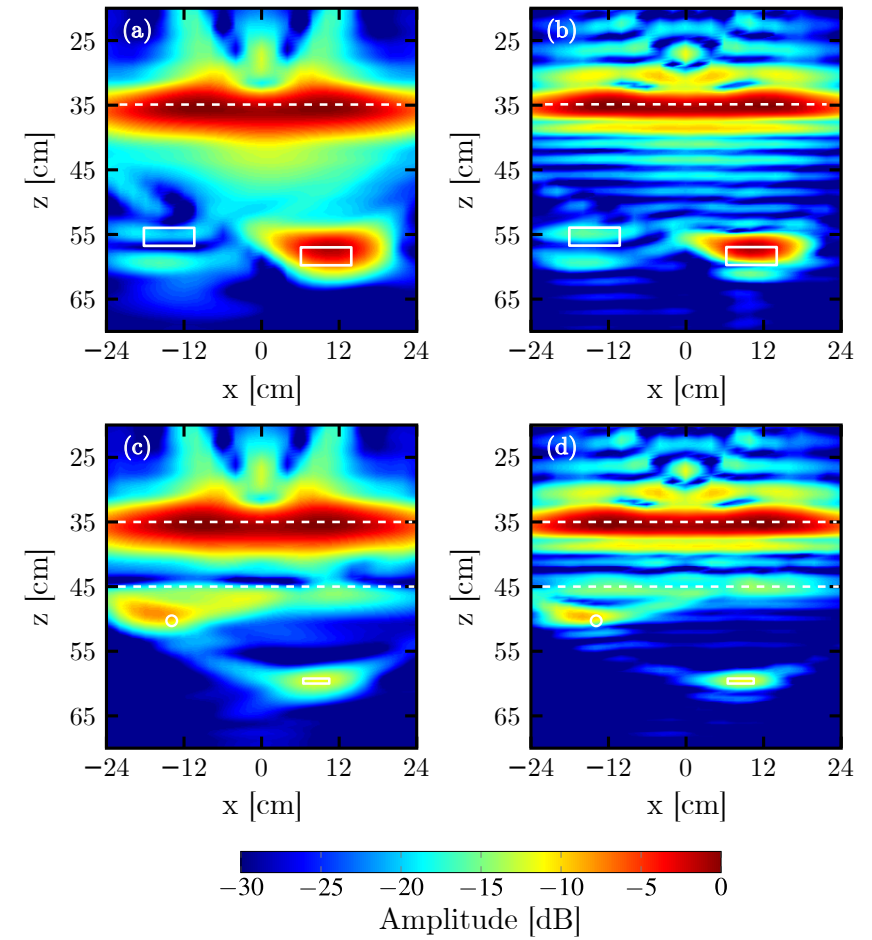

Fig. 11. Reflectivity DAS multilayer images (from simulations using a model of real bow-ties antennas). (a) Scenario A, without equalization. (b) Scenario A, with equalization. (c) Scenario B, without equalization. (d) Scenario B, with equalization.

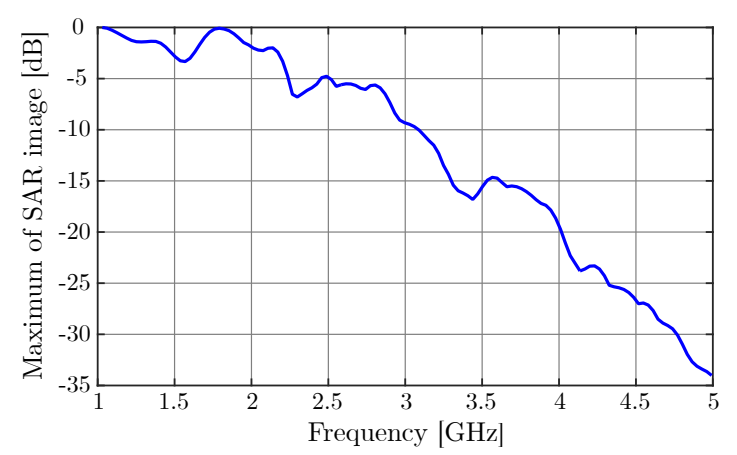

Fig. 12. Maximum of SAR image per frequency in Scenario B.

not applied, this interface and the cylindric object are more blurred. For this scenario, the maximum of the SAR images retrieved for each individual frequency is shown in Fig. 12. It can be noticed that the maximum decays with frequency. Thus, higher frequencies are masked by lower ones, and the effective range resolution is smaller than the theoretical one, as observed in Fig. 11 (c). Once the equalization of the frequency response is applied, range resolution increases (Fig. 11 (d)). Quantitatively, the range resolution obtained with and without equalization can be compared. This range resolution has been estimated calculating the $3-\mathrm{dB}$ range-width of the air - soil interface, which is $3.9 \mathrm{~cm}$ without equalization and $2.7 \mathrm{~cm}$ with equalization. Nevertheless, the improvement is more clearly observed comparing the reflectivity images qualitatively.

As explained before, the propagation of EM waves, the 


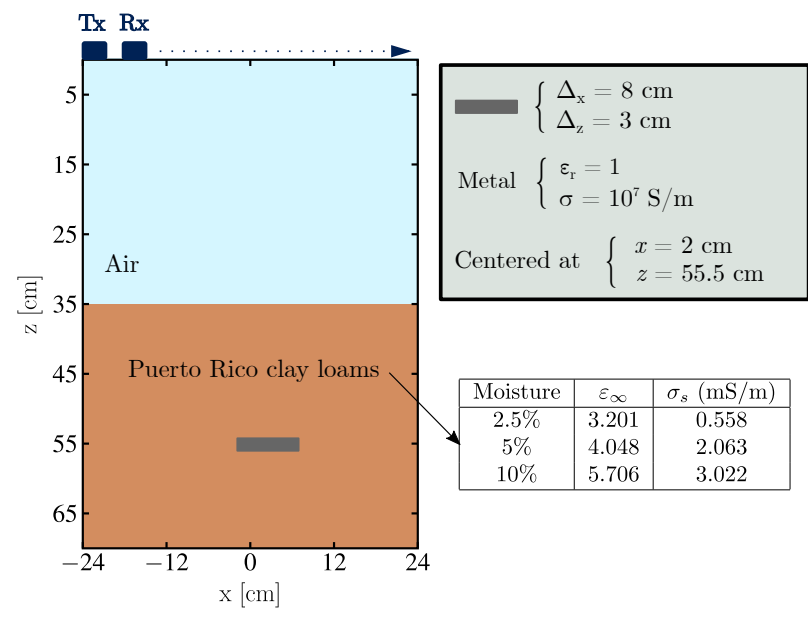

Fig. 13. Simulated Scenario C.

constitutive parameters of the materials and the behaviour of the antennas and RF equipment are frequency dependent. Therefore, some targets can be better detected considering only some frequencies (a sub-band) instead of the whole frequency band. To illustrate this effect, the scenario shown in Fig. 13 has been simulated considering a more realistic soil (Puerto Rico clay loams) with different levels of moisture. As at the beginning of this subsection, Hertzian dipole antennas have been used, transmitting the first derivative of a gaussian pulse centered at $3 \mathrm{GHz}$. This soil has been modeled using a two-term Debye model [28] so as to take into account its frequency-dependent behaviour. The relative permittivity at $f=\infty\left(\varepsilon_{\infty}\right)$ and the static electric conductivity $\left(\sigma_{s}\right)$ are shown in Fig. 13, and the parameters for the Debye poles are given in Table 1 of [28].

Three different frequency bands have been considered: $1-6 \mathrm{GHz}$ (corresponding to approximately the whole useful bandwidth), $1-3 \mathrm{GHz}$ and $4-6 \mathrm{GHz}$. The reflectivity images considering a $5 \%$ moisture are shown in Fig. 14. The targets exhibits higher reflectivity when considering the low frequency sub-band. In this simulation, it is clear that there is a buried target at around $54 \mathrm{~cm}$. However, in real measurements some targets might be almost indistinguishable from the clutter (especially, when the soil is heterogeneous and lossy). In these cases, selecting a sub-band could help to decide whether there is a target or not.

In order to quantitatively assess the effect of selecting a subband, the difference between the reflectivity of the air - soil interface and of the target is given in Table I. As the soil losses increase, this difference also increases (being more difficult to detect the target). In this example, it must be noticed that this difference is greater when considering the high frequency sub-band, and smaller for the low one. Thus, the target can be better distinguished using the lower frequencies (at the expense of worse resolution because of using smaller bandwidth).

Regarding the use of image domain partitioning, the beamwidth of the antennas that are available in the simulation software is around $90^{\circ}$, so the antennas illuminate almost the whole imaging domain. In addition, the antenna patterns
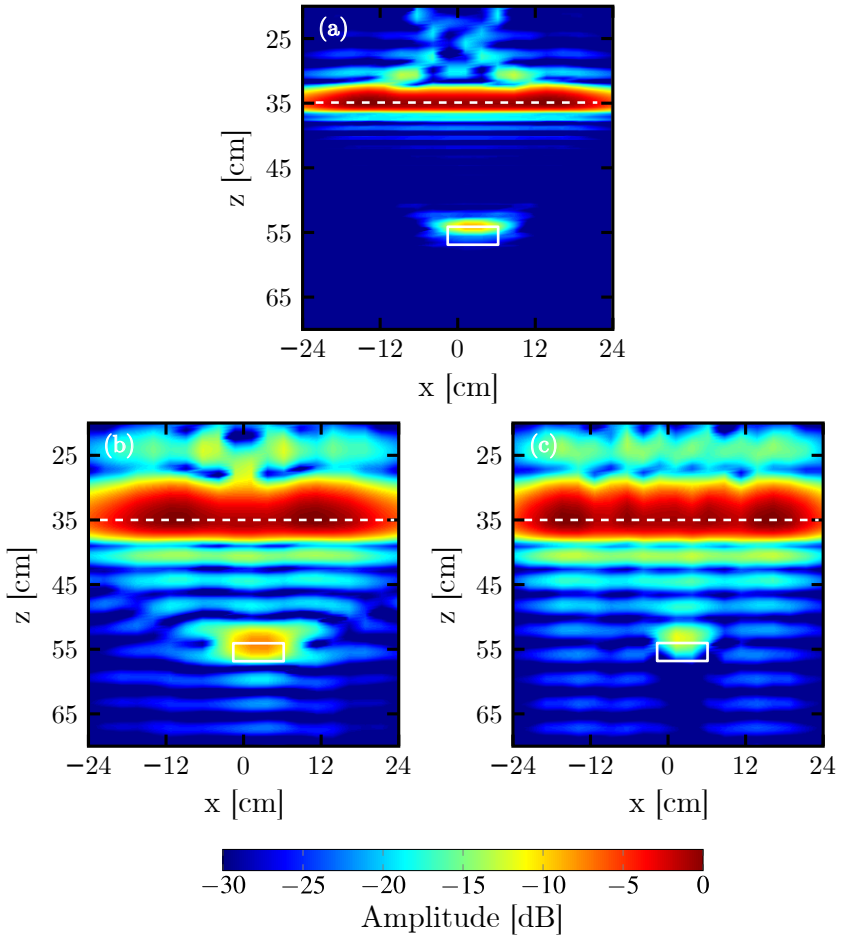

Fig. 14. Reflectivity DAS multilayer images of Scenario C considering $5 \%$ moisture. Frequency band: $1-6 \mathrm{GHz}(\mathrm{a}), 1-3 \mathrm{GHz}(\mathrm{b})$, and $4-6 \mathrm{GHz}$ (c).

TABLE I

SAR REFLECTIVITY DIFFERENCE BETWEEN THE AIR - SOIL INTERFACE AND THE TARGET (IN DB).

\begin{tabular}{|c|c|c|c|c|}
\cline { 3 - 5 } \multicolumn{2}{c|}{} & \multicolumn{3}{c|}{ Frequency band } \\
\cline { 3 - 5 } \multicolumn{2}{c|}{} & $1-6 \mathrm{GHz}$ & $1-3 \mathrm{GHz}$ & $4-6 \mathrm{GHz}$ \\
\hline \multirow{3}{*}{ Moisture } & $2.5 \%$ & -0.1 & -0.1 & 1.6 \\
\cline { 2 - 5 } & $5 \%$ & 9.2 & 7.9 & 11.9 \\
\cline { 2 - 5 } & $10 \%$ & 13.4 & 11.6 & 15.7 \\
\hline
\end{tabular}

at each single frequency cannot be obtained (a measure of the total energy is given instead). Therefore, image domain partitioning cannot be applied to the simulations. The result of this technique will be shown in the measurements. It must be remarked that the benefits of all these improvements are more evident when processing the measurements, mainly due to the stronger frequency dependent behaviour of both the equipment and the inspected soil, as well as the soil heterogeneity.

\section{B. Measurement in a controlled environment}

The proposed techniques have been validated using a $X Y Z$ measurement range [29], which has been modified to enable quasi-monostatic measurements. A N5244A PNA-X Vector Network Analyzer (VNA) [30] calibrated from $0.4 \mathrm{GHz}$ to $7 \mathrm{GHz}$ has been used together with the two Vivaldi antennas previously described [25] to measure the transmission coefficient $\left(S_{21}\right)$. The $S_{21}$ parameter is directly proportional to the scattered field, so DAS and PSM algorithms can be applied exactly as if the scattered field were acquired. The VNA has been calibrated at the edges of the coaxial cables connecting the VNA ports and the Vivaldi antennas, 


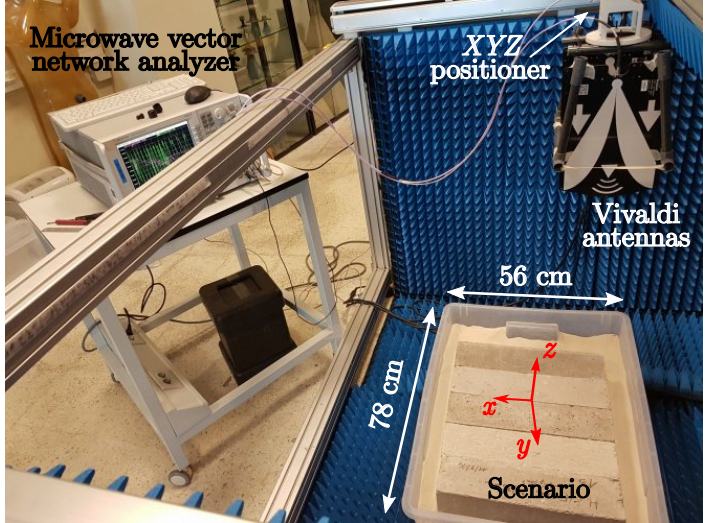

Fig. 15. Picture of the $X Y Z$ measurement range [29] configured for quasimonostatic GPR measurements.

so the effects of the cables and connectors are removed from the measurements. The $X Y Z$ measurement range is covered with APM9 pyramidal absorber [31], which provides $-15 \mathrm{~dB}$ reflectivity at $1 \mathrm{GHz}$, and $-29 \mathrm{~dB}$ reflectivity at $4 \mathrm{GHz}$.

A rectangular acquisition grid of size $x=70 \mathrm{~cm}, y=$ $80 \mathrm{~cm}$ sampled every $\delta x=\delta y=2 \mathrm{~cm}$ (resulting in 1476 measurement points) and placed at $z=120 \mathrm{~cm}$ above the floor of the $X Y Z$ measurement facility, has been considered. For this setup, measurement time is around 50 minutes. A scheme of the measurement setup is shown in Fig. 15.

The first measurement scenario is depicted in Fig. 16. It is composed by a rectangular plastic box filled with dry sand $\left(\varepsilon_{r}=2.5\right)$ and two objects (metallic and plastic disks) buried at different depths. The absorbing material under the plastic box had to be removed, so the plastic box was placed on the metallic floor of the $X Y Z$ measurement range.

DAS and PSM multilayer results, considering dry sand permittivity, are plotted in Fig. 17 without applying any of the improvements described in Section III. The following reflections can be identified in the reflectivity images: the airsand interface $(z=0.98 \mathrm{~m})$, the plastic disk $(z=1.06 \mathrm{~m})$, the metallic disk $(z=1.10 \mathrm{~m})$, and the sand-metallic floor interface $z=1.30 \mathrm{~m}$. Besides, multiple reflections occur due to the finite size of the plastic box filled with sand, the air-sand impedance mismatching and the high reflectivity of the metallic floor, which result in the echo appearing at $z=1.44 \mathrm{~m}$.

Amplitude differences between low and high frequency bands have different impact in PSM and DAS. In particular, less clutter is observed in PSM images. Regarding SAR image resolution (i.e. visualization of the interfaces between different media and reconstruction of the buried objects profile), both techniques perform similarly.

Some improvements are introduced in order to mitigate the clutter presence in DAS algorithm, which can be applied to PSM algorithm as well. First, sub-band processing is tested. Taking into account frequency dependence of propagation losses and directivity of the selected antennas [25], as discussed in Section III, it can be expected that lower subbands present more penetration capabilities, whereas higher sub-bands exhibit better resolution. These hypotheses are

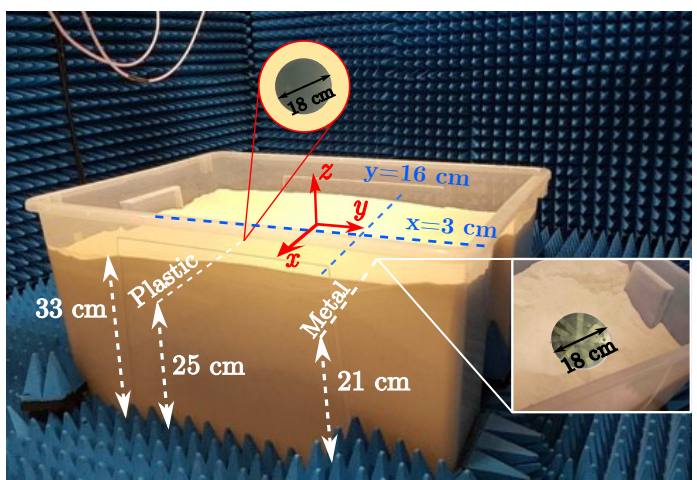

Fig. 16. Measurement scenario consisting of two buried objects (metallic and plastic) buried in a plastic box filled with dry sand.
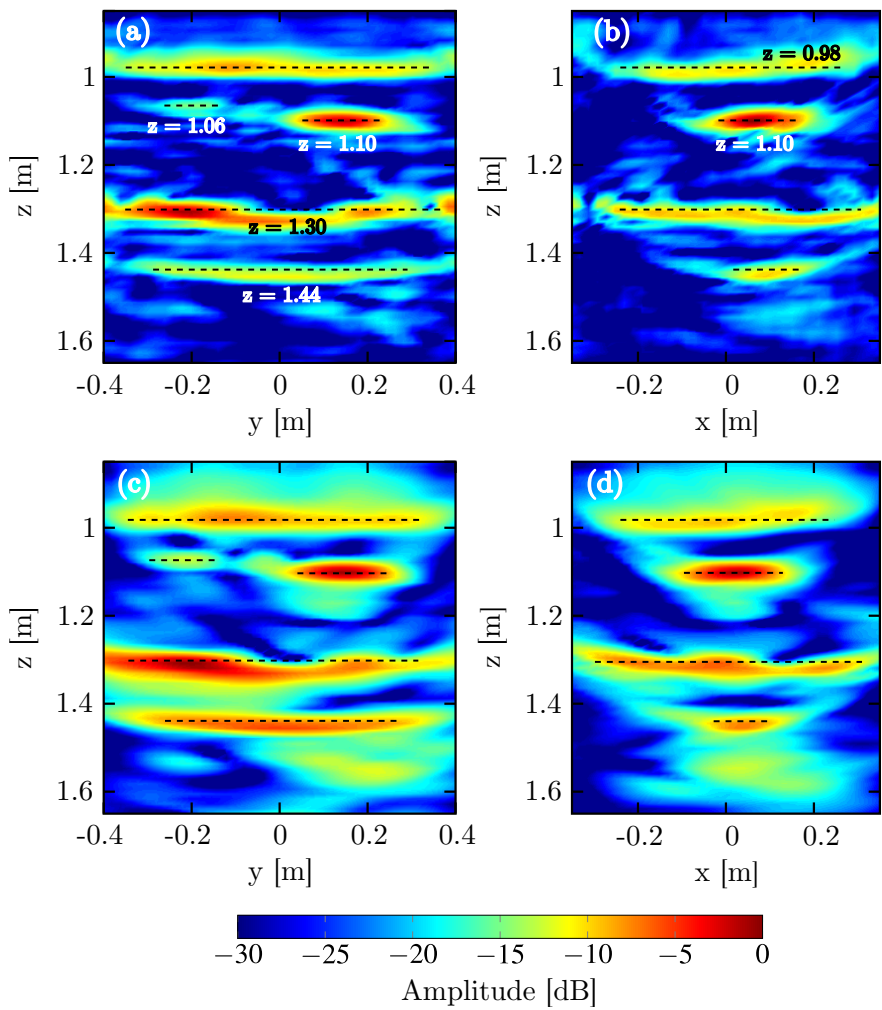

Fig. 17. Reflectivity images, $Y Z$ plane at $x=3 \mathrm{~cm}$ and $X Z$ plane at $y=16$ $\mathrm{cm}$ of the scenario depicted in Fig. 16. (a)-(b) PSM multilayer. (c)-(d) DAS multilayer.

consistent with the results shown in Fig. 18: (a)-(b) correspond to the low frequency sub-band (1-3 GHz), and (c)-(d) to the high frequency sub-band $(4-6 \mathrm{GHz})$.

It can be noticed that lower frequency sub-band results (Fig. 18 (a)-(b)) are similar to those obtained considering the entire band (Fig. 17 (c)-(d)). This is due to the fact that, as discussed in Section III, the frequency response of the GPR system at lower frequencies is higher, thus masking the response at higher frequencies. For this reason, the improvement consisting of the equalization of the frequency response is applied, resulting in greater range resolution and less clutter, as observed in Fig. 18 (e)-(f).

In a second scenario, five $10 \mathrm{~cm}$ thick blocks of concrete 

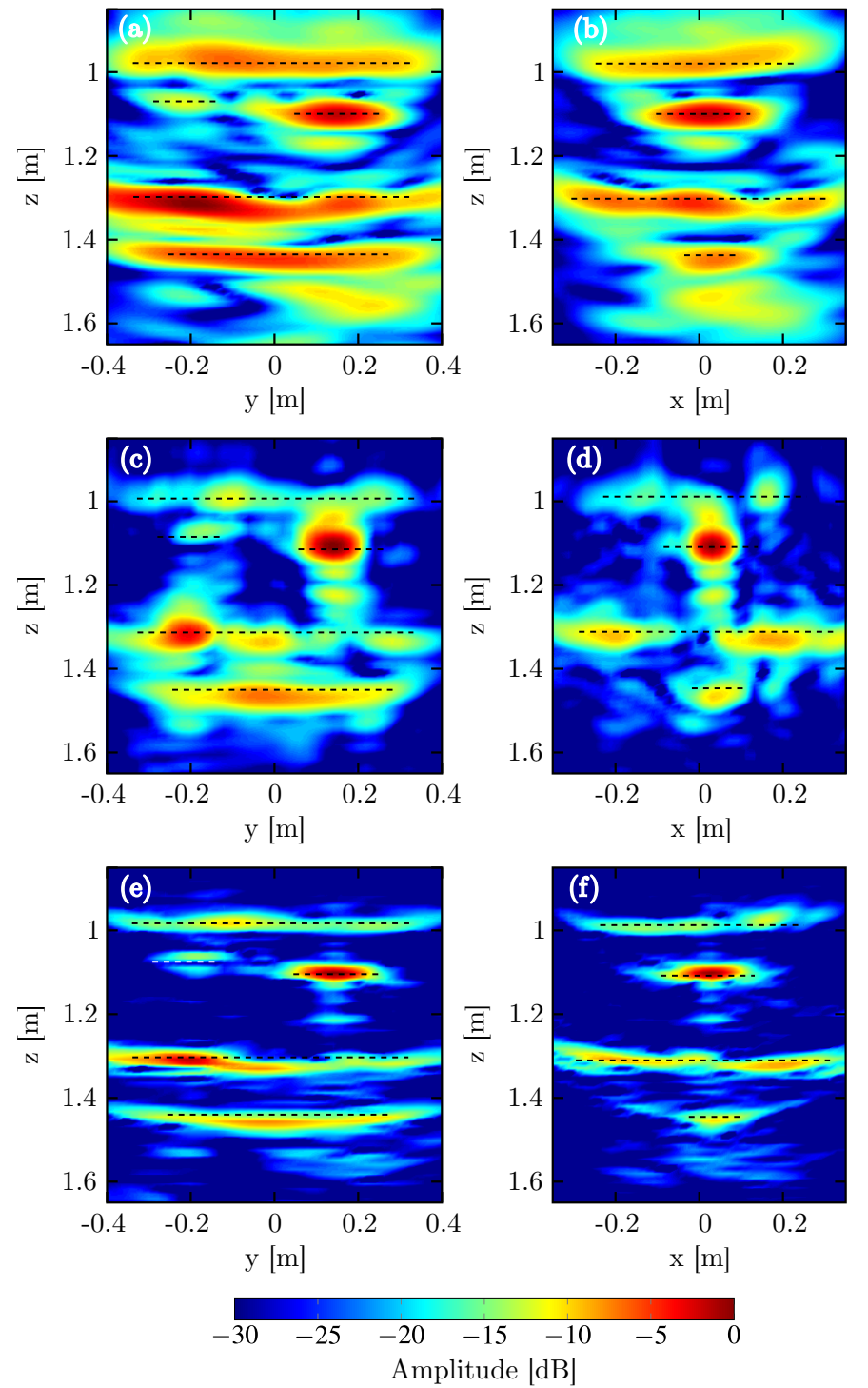

Fig. 18. Reflectivity DAS multilayer images representing $Y Z$ plane at $x=3$ $\mathrm{cm}$ and $X Z$ plane at $y=16 \mathrm{~cm}$ of the scenario depicted in Fig. 16. (a)-(b) Sub-band processing ( 1 to $3 \mathrm{GHz}$ ). (c)-(d) Sub-band processing (4 to $6 \mathrm{GHz}$ ). (e)-(f) Equalization of the frequency response.

$\left(\varepsilon_{r}=9\right)$ have been placed on the dry sand layer. A metallic disk is buried at $10 \mathrm{~cm}$ depth under the concrete-sand interface (i.e. under the concrete blocks), as depicted in Fig. 19. First, DAS and PSM multilayer techniques were applied without any improvement technique (as in the previous measurement scenario). Results are depicted in Fig 20, where the following interfaces can be identified: the air-concrete interface at $z=$ $0.88 \mathrm{~m}$, the air-sand interface at $z=0.92 \mathrm{~m}$ (this is the area not covered with concrete blocks), the concrete-sand interface at $z=0.99 \mathrm{~m}$ (consistent with the $10 \mathrm{~cm}$ thickness of the concrete blocks), the buried object at $z=1.11 \mathrm{~m}$ (PSM) or $z=1.09 \mathrm{~m}$ (DAS) and the sand-ground plate interface at $z=$ $1.18 \mathrm{~m}$ (reflections through sand) and $z=1.30 \mathrm{~m}$ (reflections through concrete blocks and sand).

As in the previous measurement example, improvement techniques developed in Section III are needed to increase the image resolution. Here, imaging domain partitioning is

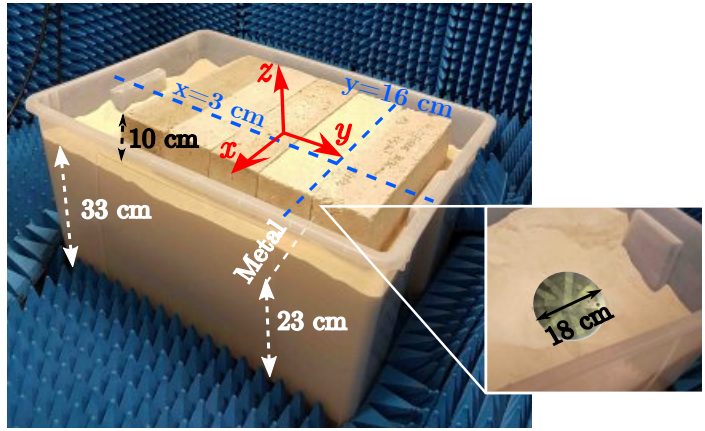

Fig. 19. Measurement scenario consisting of a $10 \mathrm{~cm}$ concrete layer on top of a $33 \mathrm{~cm}$ dry sand layer, where a metallic disk is buried.

introduced together with the equalization of the frequency response. For this example, cell size is $2 \mathrm{~cm}$, the same as the sampling rate of the acquisition domain grid. As explained in Section III, the number of cells is chosen as a function on the antenna beamwidth. Analysing Fig.5 it can be observed that the antenna beamwidth for frequencies below $2 \mathrm{GHz}$ is greater than 50 degrees. That means that, for $f=2 \mathrm{GHz}$ and at $h=1 \mathrm{~m}$ from the antenna (where the air - sand interface is located) the number of cells to be considered is (Eq. 10) $C_{x, n}=31$ and $C_{y, n}=45$, i.e. an area of $62 \mathrm{~cm} \times 90 \mathrm{~cm}$. As a result, at low frequencies the whole area of the plastic box is fully illuminated by the beam of the antennas when these are centered over it.

Results for DAS algorithm are depicted in Fig. 21: (a)-(b) correspond to the equalization of frequency response, and (c)(d) to both equalization and imaging domain partitioning. It can be observed that results are not improved significantly when introducing imaging domain partitioning, due to the use of antennas with broad beam at low frequencies together with the finite size of the scenario, which is fully covered by the beam for central positions of the scanning grid. Nevertheless, imaging domain partitioning reduces the calculation time as the backpropagated scattered field has to be evaluated in less points of the imaging domain (i.e. those corresponding to the selected cells for each frequency). For this example, calculation time is reduced around $25 \%$.

In this example it can be noticed that equalization of the frequency response to increase range resolution results in worse penetration capabilities. If Fig. 20, and Fig. 21 are compared, the metallic disk buried in sand is better detected in Fig 20 (for both DAS and PSM), where equalization of the frequency response was not applied. Low frequencies, capable of penetrating better in the concrete layer, contribute more to the radar image than high frequencies.

\section{Measurement in a realistic scenario}

Finally, the proposed techniques have been validated in an outdoor scenario. A portable setup has been deployed in a beach (coordinates 43.545, -5.694) close to the laboratory of the research group. A radar module [24] working from 100 $\mathrm{MHz}$ to $6.5 \mathrm{GHz}$ connected to a Raspberry Pi controller and the previous Vivaldi antennas [25] (as transmitter and receiver antennas) have been used. Measurements have been done by 

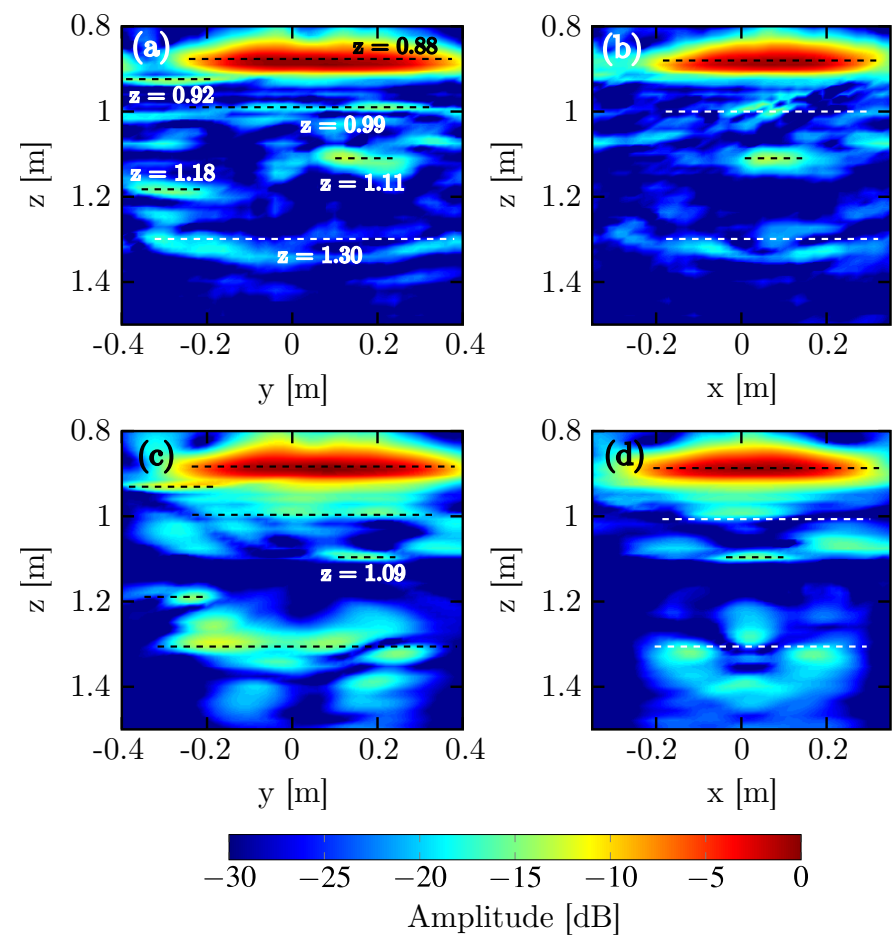

Fig. 20. Reflectivity images representing $Y Z$ plane at $x=3 \mathrm{~cm}$ and $X Z$ plane at $y=16 \mathrm{~cm}$ of the scenario depicted in Fig. 19. (a)-(b) PSM multilayer. (c)-(d) DAS multilayer.
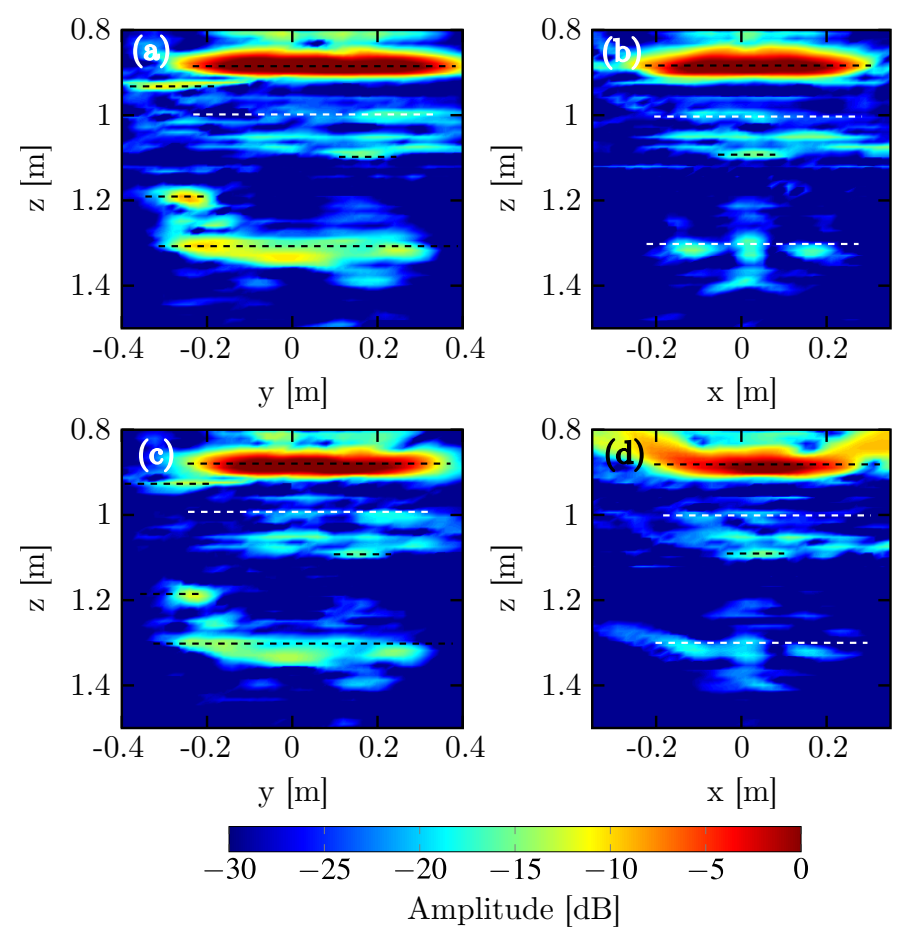

Fig. 21. Reflectivity images representing $Y Z$ plane at $x=3 \mathrm{~cm}$ and $X Z$ plane at $y=16 \mathrm{~cm}$ of the scenario depicted in Fig. 19. (a)-(b) DAS multilayer, equalization of the frequency response. (c)-(d) DAS multilayer, equalization and imaging domain partitioning.

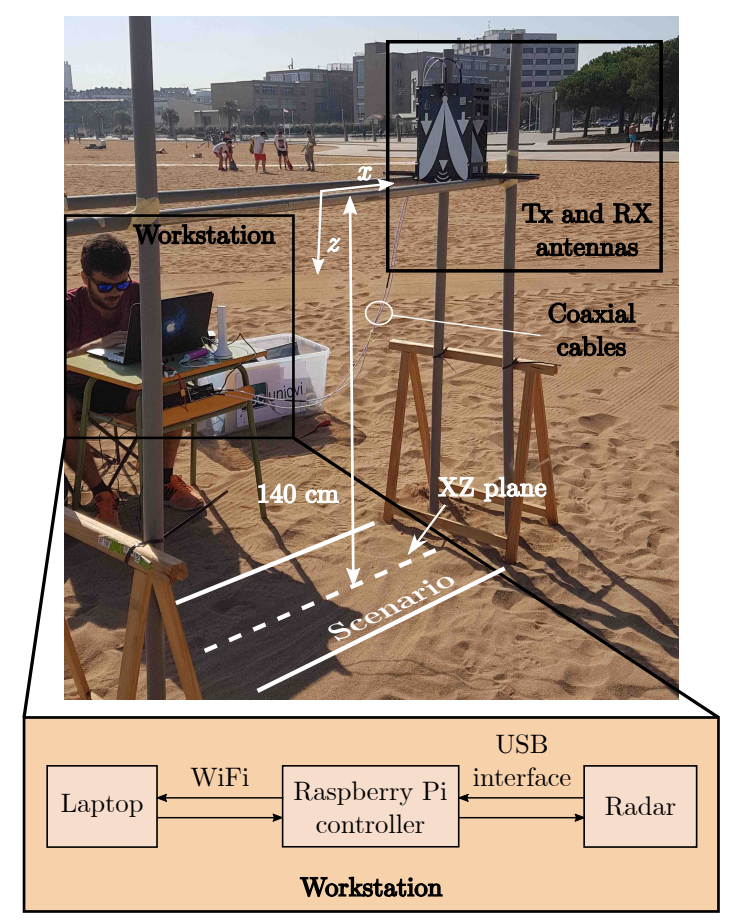

Fig. 22. Portable GPR measurement setup deployed in a beach.

manually sweeping the Vivaldi antennas every $2 \mathrm{~cm}$ along a $120 \mathrm{~cm}$ slider made of plastic pipes, and placed $140 \mathrm{~cm}$ above the ground, as depicted in Fig. 22. GPR measurements were sent to a laptop using a WiFi connection, for later processing.

Concerning protection against electromagnetic interference that might occur in realistic scenarios, it must be remarked that the power transmitted by the radar module $(-10 \mathrm{dBm})$ is expected to be higher than the level of potential interfering signals (such as those coming from broadcasting and mobile communications networks, for example). Moreover, the proposed GPR is an ultrawideband system (ranging from less than $1 \mathrm{GHz}$ to up to $6 \mathrm{GHz}$ ). Thus, even high power narrowband signals will have little impact in the measured response of the radar, as it is integrated over the full bandwidth. Actually, robustness against narrowband interference is one of the main advantages of ultrawideband communications systems. Finally, it must be remarked that the Tx and Rx antennas are pointing towards the ground (downward-looking configuration) which reduces even more the possibility of capturing an interfering signal.

The sand of the beach was compacted by sea moisture, so it would be altered every time a hole is dug to bury an object. For this reason, a homogeneous volume of sand was created for the experiments by turning over the sand up to a depth of 25 $\mathrm{cm}$. Next, two objects were buried in this homogeneous layer of sand: an empty plastic container and a metallic disk (Fig. 23 (b)). Imaging results for multilayer processing are shown in Fig. 24, considering $\varepsilon_{r}=3$ for the sand of the beach. White dashed lines represent the cross-section of the limits of the ditch filled with sand, whereas black dotted lines indicate the placement and size of the buried targets.

Taking into account that i) the antennas were manually moved and ii) the air-sand interface is not perfectly flat, higher 

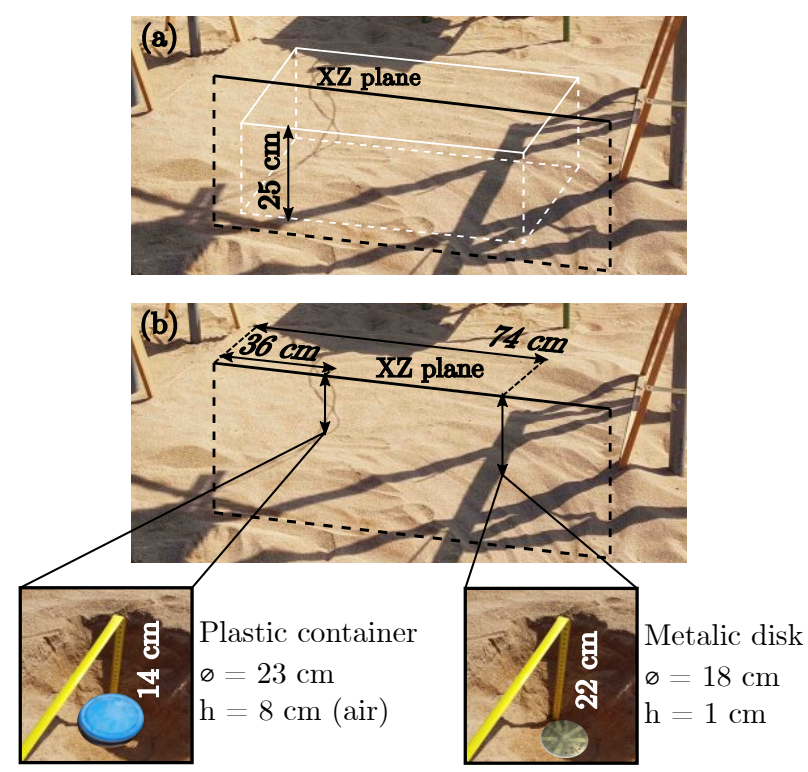

Fig. 23. Tested scenarios: (a) Ditch filled with sand. (b) Ditch filled with sand, with two buried objects (metallic and plastic).

levels of noise and clutter than in previous examples are expected. DAS results with no improvements are depicted in Fig. 24 (a),(d). Although stronger reflections at $z=56 \mathrm{~cm}$ and $z=64 \mathrm{~cm}$ can be observed in Fig. 24 (d), it is not possible to clearly distinguish the buried objects.

Next, equalization of the frequency response has been applied in Fig. 24 (b),(e). In the case of Fig. 24 (e), the reflection on the buried metallic disk can be clearly identified, but not the plastic container because its reflectivity is similar to the clutter level.

Finally, GPR-SAR processing, considering both equalization of the frequency response and imaging domain partitioning, is applied. In this case, $h=140 \mathrm{~cm}$, so the number of cells to be considered in the $X Z$ plane for $f=2 \mathrm{GHz}$ is (Eq. 10) $C_{x, n}=63$. In other words, the antenna beam illuminates $\pm 63 \mathrm{~cm}$ along the $x$ axis with respect to the position of the antennas.

Imaging results are depicted in Fig. 24 (c),(f), where the reduction of clutter can be noticed. In this case, both metallic and plastic objects can be distinguished from the remaining clutter. Furthermore, the reflection on the interface between the homogeneous sand in the ditch and the compact wet sand can be noticed at $z=67 \mathrm{~cm}$ in Fig. 24 (c) and partially in Fig. 24 (f) under the plastic object.

\section{CONCLUSION}

Three methodologies have been proposed for improving UWB GPR-SAR-based techniques, especially for those measurement setups where parameters such as frequency response and antenna directivity have a strong variation with frequency. It has been proved that the first one, equalization of the frequency response, allows increasing range resolution as the same weight is assigned to low and high frequencies contributing to the SAR image, but at the expense of worsening ground penetration. The second method, sub-band processing, takes advantage of the properties of each frequency sub-band (better ground penetration or better resolution) but cannot create a single SAR image that combines the advantages of each subband. Finally, imaging domain partitioning takes into account antenna beamwidth to reduce the number of points of the imaging domain where the backpropagated field has to be computed, thus reducing both the clutter and the calculation time.

Depending on the characteristics of the area to be surveyed and the specifications of the GPR system, these improvement techniques may be combined and applied in UWB GPRSAR systems for an accurate detection of buried metallic and non-metallic targets. For example, in a controlled scenario such as the one presented in Section IV-B, even at lower frequency bands it is possible to achieve sufficient resolution to distinguish the buried targets (as shown in Fig. 20). The use of equalization technique improves range resolution, but at the expense of worsening the reflectivity of the buried targets (Fig. 21). However, in a realistic scenario as the one shown in Fig. 24 , if improvement techniques are not applied, it is almost impossible to distinguish anything due to the higher level of clutter and noise. The improvement in the reflectivity image after the equalization of the frequency response (and imaging domain partitioning) clearly makes the use of these techniques necessary to have enough resolution to distinguish the objects in harsh environments.

\section{ACKNOWLEDGEMENT}

The authors would like to thank Prof. Fernando López Gayarre from the Department of Construction and Manufacturing Engineering at University of Oviedo for supplying the concrete blocks used in the second measurement setup of Section IV-B.

\section{REFERENCES}

[1] H. Hold, Ground Penetrating Radar: Theory and Applications. Amsterdam: Elsevier Science, 2008.

[2] J. S. Lee, C. Nguyen, and T. Scullion, "A novel, compact, lowcost, impulse ground-penetrating radar for nondestructive evaluation of pavements," IEEE Transactions on Instrumentation and Measurement, vol. 53, pp. 1502-1509, 2004.

[3] P. Klinkhachorn, A. S. Mercer, U. B. Halabe, and H. G. Rao, "An autonomous unmanned ground vehicle for nondestructive testing of fiber-reinforced polymer bridge decks," IEEE Instrumentation and Measurement Magazine, vol. 10, pp. 228-233, 2007.

[4] J. A. Martinez-Lorenzo, C. M. Rappaport, and F. Quivira, "Physical limitations on detecting tunnels using underground-focusing spotlight synthetic aperture radar," IEEE Transactions on Geoscience and Remote Sensing, vol. 49, pp. 65-70, 2011.

[5] R. Siegel, "Land mine detection," IEEE Instrumentation and Measurement Magazine, vol. 5, pp. 22-28, 2002.

[6] D. J. Daniels, "A review of GPR for landmine detection," Sensing and Imaging: an International Journal, vol. 7, pp. 90-123, 2006.

[7] B. Gonzalez, Y. Alvarez, A. Arboleya, Y. R. Vaqueiro, M. Garcia, F. LasHeras, and A. G. Pino, "Airborne systems and detection methods localization and production of images of buried objects and characterization of the composition of the subsurface," https://goo.gl/JjN2bH, 72017 , patent PCT/ES2017/000006.

[8] M. Garcia-Fernandez, Y. Alvarez-Lopez, A. Arboleya-Arboleya, B. Gonzalez-Valdes, Y. Rodriguez-Vaqueiro, F. Las-Heras-Andres, and A. Pino-Garcia, "Synthetic aperture radar imaging system for landmine detection using a ground penetrating radar on board an unmanned aerial vehicle," IEEE Access, vol. 6, pp. 2169-3536, 2018.

[9] M. Schartel, R. Burr, W. Mayer, N. Docci, and C. Waldschmidt, "UAV-based ground penetrating synthetic aperture radar," in 2018 IEEE MTT-S International Conference on Microwaves for Intelligent Mobility (ICMIM), 2018, pp. 1-4. 

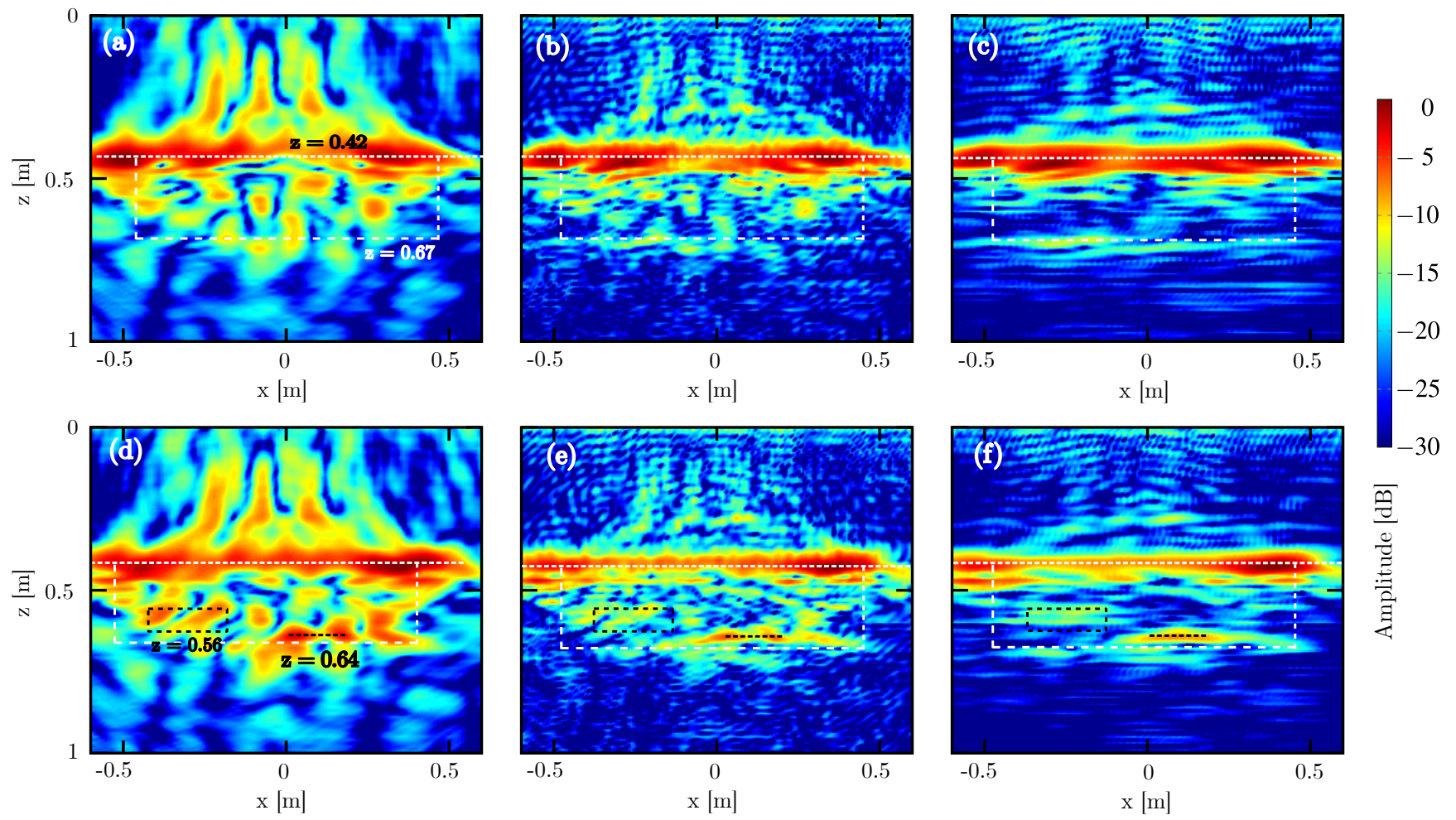

Fig. 24. Reflectivity multilayer images ( $X Z$ plane). First row corresponds to the scenario of Fig. 23 (a) and second row, to the scenario of Fig. 23 (b). (a),(d) No improvements. (b),(e) Equalization. (c),(f) Equalization and imaging domain partitioning.

[10] J. T. Case, M. T. Ghars, and R. Zoughi, "Optimum two-dimensional uniform spatial sampling for microwave SAR-based NDE imaging systems," IEEE Transactions on Instrumentation and Measurement, vol. 60, pp. 3806-3815, 2011.

[11] M. Llort, A. Aguasca, C. Lopez-Martinez, and T. Martinez-Marin, "Initial evaluation of SAR capabilities in UAV multicopter platforms," IEEE Journal of Selected Topics in Applied Earth Observations and Remote Sensing, vol. 11, pp. 127-140, 2018

[12] M. Pastorino, S. Caorsi, and A. Massa, "A global optimization technique for microwave nondestructive evaluation," IEEE Transactions on Instrumentation and Measurement, vol. 51, pp. 666-673, 2002.

[13] Y. Alvarez, M. Garcia-Fernandez, L. Poli, C. Garcia-Gonzalez, P. Rocca, A. Massa, and F. Las-Heras, "Inverse scattering for monochromatic phaseless measurements," IEEE Transactions on Instrumentation and Measurement, vol. 66, pp. 45-60, 2017.

[14] Y. Alvarez, M. Garcia, A. Arboleya, B. Gonzalez, Y. R. Vaqueiro, F. Las-Heras, and A. G. Pino, "SAR-based technique for soil permittivity estimation," International Journal of Remote Sensing, vol. 38, pp. 51685186, 2017.

[15] E. M. Johansson and J. E. Mast, "Three-dimensional ground-penetrating radar imaging using synthetic aperture time-domain focusing," in SPIE's 1994 International Symposium on Optics, Imaging, and Instrumentation, International Society for Optics and Photonics, 1994, pp. 205-214.

[16] D. H. N. Marpaung and Y. Lu, "A comparative study of migration algorithms forUWB GPR images in SISO-SAR and MIMO-array configurations," in 15th IEEE International Radar Symposium (IRS), 2014, pp. $1-4$.

[17] C. Ozdemir, S. Demirci, E. Yigit, and B. Yilmaz, "A review of migration methods in b-scan ground penetrating radar imaging," Mathematical Problems in Engineering, vol. 2014, pp. 1-16, 2014.

[18] M. Fallahpour, J. T. Case, M. T. Ghasr, and R. Zoughi, "Piecewise and wiener filter-based SAR techniques for monostatic microwave imaging of layered structures," IEEE Transactions on Antennas and Propagation, vol. 62, pp. 282-294, 2014.

[19] M. Fallahpour and R. Zoughi, "Sensitivity analysis of wiener-filterbased synthetic aperture radar (SAR) microwave imaging technique," in Proceeding of the 2014 IEEE International Instrumentation and Measurement Technology Conference (I2MTC), 2014, pp. 1-4.
[20] F. Abujarad, G. Nadim, and A. Omar, "Clutter reduction and detection of landmine objects in ground penetrating radar data using singular value decomposition (SVD)," in Proc. of the 3rd International Workshop on Advanced Ground Penetrating Radar (IWAGPR), 2005, pp. 1-4.

[21] M. Garcia-Fernandez, Y. Alvarez-Lopez, Y. Rodriguez-Vaqueiro, B. Gonzalez-Valdes, A. Arboleya-Arboleya, F. Las-Heras, and A. PinoGarcia, "SVD-based clutter removal technique for GPR," in 2017 IEEE AP-S Symposium on Antennas and Propagation, 2017, pp. 2369-2370.

[22] R. Solimene, A. Cuccaro, A. Dell'Aversano, I. Catapano, and F. Soldovieri, "Ground clutter removal in GPR surveys," IEEE Journal of Selected Topics in Applied Earth Observations and Remote Sensing, vol. 7, pp. 792-798, 2014.

[23] J. F. Claerbout, Imaging the Earth's Interior. Stanford University, 1984.

[24] $(2018,10) \mathrm{m}$ :explore UWB radar from ILMSense. [Online]. Available: https://www.uwb-shop.com/products/m-explore/

[25] $(2018,10)$ UWB antenna from RFSPACE. [Online]. Available: http://www.rfspace.com/RFSPACE/Antennas.html

[26] Q. Li, Z. Xia, J. Shao, J. Chen, X. Liu, and G. Fang, "Non-destructive survey of pavement layer thicknesses with ground penetrating radar," in 2013 IEEE International Conference of IEEE Region 10 (TENCON 2013), Oct 2013, pp. 1-4.

[27] C. Warren, A. Giannopoulos, and I. Giannakis, "gprmax: Open source software to simulate electromagnetic wave propagation for ground penetrating radar," Computer Physics Communications, vol. 209, pp. 163-170, 2016.

[28] "A complete fdtd simulation of a real gpr antenna system operating above lossy and dispersive grounds," Progress in Electromagnetics Research, vol. 50, pp. 209-229, 2005.

[29] A. Arboleya, Y. Alvarez, and F. Las-Heras, "Millimeter and submillimeter planar measurement setup," in 2013 IEEE AP-S Symposium on Antennas and Propagation, 2013, pp. 1-2.

[30] $(2018,10)$ N5247a PNA-x microwave network analyzer from Keysight. [Online]. Available: https://www.keysight.com/en/ pdx-x201825-pn-N5247A/pna-x-microwave-network-analyzer-67-ghz? $\mathrm{cc}=\mathrm{EN} \& \mathrm{lc}=\mathrm{eng}$

[31] $(2019,3)$ APM9 microwave pyramidal absorber from Siepel. [Online]. Available: https://www.hik-consulting.pl/files/ microwave-pyramidal-absorber-apm---01-02-12.pdf 